\title{
INVESTIGACIONES EN SALUD SEXUAL Y REPRODUCTIVA DE PUEBLOS INDÍGENAS EN CHILE Y LA AUSENCIA DE PERTINENCIA ÉTNICA
}

\author{
Claudia Dides Castillo* y María Soledad Pérez Moscoso**
}

\begin{abstract}
Resumen: El artículo reflexiona sobre la pertinencia étnica de las investigaciones desarrolladas en Chile sobre salud sexual y reproductiva entre 1990 y 2004, basándose en un diagnóstico nacional sobre el tema. Se constata que la mayoría de estas investigaciones no consideraron la diversidad cultural y étnica de nuestro país, siendo éste un tema pendiente. A su vez, se plantean las tensiones que implica la ausencia de pertinencia étnica en las investigaciones en salud sexual y reproductiva.
\end{abstract}

Palabras clave: pueblos indígenas, salud sexual y reproductiva, pertinencia étnica

\section{INVESTIGATIONS ON REPRODUCTIVE AND SEXUAL HEALTH IN CHILEAN INDIGENOUS POPULATIONS AND THE LACK OF ETHNIC RELEVANCE}

\begin{abstract}
This essay argues about the ethnic relevance of investigations carried out in Chile on sexual and reproductive health from 1990 to 2004, based on a national diagnosis on the subject. Most of these investigations did not consider the cultural and ethnic diversity of our country, so this is a pending issue. Furthermore, the tensions implied by the lack of ethnic relevance in investigations about sexual and reproductive health are also raised..
\end{abstract}

Key words: indigenous populations, sexual and reproductive health, ethnic relevance

PESQUISAS EM SAÚDE SEXUAL E REPRODUTIVA DOS POVOS INDÍGENAS NO CHILE E A AUSÊNCIA DE PERTINÊNCIA ÉTNICA

Resumo: O artigo reflete sobre a pertinência étnica das pesquisas desenvolvidas no Chile sobre a saúde sexual e reprodutiva entre 1990-2004, baseando-0se num diagnóstico nacional sobre o tema. Constata-se que a maioria destas pesquisas não levou em conta a diversidade cultural e étnica de nosso país, sendo este um tema pendente. Por sua vez, apresentam-se as tensões que implicam na ausência de pertinência étnica nas pesquisas em saúde sexual e reprodutiva.

Palavras-chave: povos indígenas, saúde sexual e reprodutiva, pertinência étnica

Socióloga. Programa Equidad de Género y Políticas Públicas, Facultad Latinoamericana de Ciencias Sociales (FLACSO), Chile Correspondencia: cdides@flacso.cl

** Psicóloga. Programa Equidad de Género y Políticas Públicas, Facultad Latinoamericana de Ciencias Sociales (FLACSO), Chile Correspondencia: solep70@yahoo.es 


\section{Introducción}

La mayoría de las investigaciones desarrolladas en Chile sobre salud sexual y reproductiva han ignorado la diversidad cultural y étnica de nuestro país, siendo éste un tema pendiente. Este artículo reflexiona acerca de esta carencia, considerada como un dilema ético, basándose en un diagnóstico nacional que recopiló y sistematizó investigaciones y experiencias en salud sexual y reproductiva en pueblos indígenas en Chile entre los años 1990 y $2004^{1}$.

El concepto de salud sexual y reproductiva se ha venido desarrollando en las últimas década, en particular desde el Programa de Acción de la Conferencia Internacional de Población y Desarrollo (El Cairo 1994). Se define como "un estado general de bienestar físico, mental y social, y no de mera ausencia de enfermedades o dolencias, en todos los aspectos relacionados con el sistema reproductivo y sus funciones y procesos (...) La salud reproductiva entraña la capacidad de disfrutar una vida sexual satisfactoria y sin riesgos y de procrear, y la libertad para decidir hacerlo o no hacerlo, cuándo y con qué frecuencia”.

Esta misma Conferencia incorporó en su Programa de Acción un capítulo sobre derechos de los pueblos indígenas, con el propósito de mejorar las capacidades reproductivas, las relaciones afectivas, el medio ambiente, los derechos a la salud sexual y reproductiva, con sus diversidades como potencial. El Enlace Continental de Mujeres Indígenas, que es parte del Foro Internacional de Mujeres Indígenas, ha realizado diversas reuniones continentales para reflexionar y acordar recomendaciones a los gobiernos, a CEPAL y otros organismos de Naciones Unidas.

El Estado chileno ha suscrito los acuerdos internacionales originados en conferencias de Naciones Unidas, en particular la de Población y Desarrollo, El Cairo, 1994, y la IV Conferencia Mundial de la Mujer, Beijing, 1995. Con ellos ha elaborado políticas públicas y desarrollado diversas acciones.

1 Los datos presentados se derivan de la investigación de la Corporación de Salud y Políticas Sociales (CORSAPS), financiada por el Fondo de Población de las Naciones Unidas (UNFPA), "Diagnóstico nacional de investigaciones y experiencias en salud sexual y reproductiva en pueblos indígenas en Chile", publicada en octubre de 2005. Pérez Moscoso MS, Dides Castillo C. Salud, Sexualidad y Reproducción. Sistematización de investigaciones y experiencias en pueblos Indígenas en Chile. 1990-2004. Santiago de Chile: CORSAPS-UNFPA; 2005.
Los pueblos indígenas mantienen creencias y tradiciones que incluyen recomendaciones sobre salud sexual y reproductiva (conductas apropiadas dentro del matrimonio, sexualidad, métodos de planificación familiar), así como diversas normas y cuidados para los distintos periodos del ciclo vital reproductivo. Sin embargo, muchos de estos aspectos permanecen aún en el ámbito intracultural, siendo transmitidos a sus miembros sólo mediante procesos de socialización.

\section{Hallazgos de investigaciones sobre salud reproductiva, 1990-2004}

El acopio de información consideró las regiones de mayor concentración de población indígena en Chile, es decir, la I, VIII, IX, X regiones y la Región Metropolitana. Se incluyeron además, la II y V regiones, debido a que en ellas se localizan los pueblos indígenas Likan Antai y Rapa Nui.

Dado que el concepto salud sexual y reproductiva muchas veces aparece incomprensible para las personas y/o organizaciones indígenas, se elaboraron ejes temáticos que incluían sexualidad, regulación de la fertilidad, métodos anticonceptivos (MAC), aborto, violencia sexual, VIH/SIDA, infecciones de transmisión sexual (ITS), calidad de la atención, derechos sexuales y reproductivos, cuerpo, fecundidad, embarazo y parto, y que se transformaron en posibilidades concretas de acercamiento y comprensión mutua.

Esto permitió orientar la búsqueda de información, específicamente de referencias bibliográficas en bibliotecas, centros de documentación e Internet (catálogos en línea). Para ello se construyó una matriz cuyos principales descriptores fueron: salud sexual y reproductiva, sexualidad, género y diversas denominaciones de los pueblos indígenas ${ }^{2}$.

Una de las limitaciones de esta investigación se produjo en la etapa de recopilación de datos, debido a que no todas las fuentes bibliográficas fueron encontradas en centros de documentación, universidades, organizaciones no gubernamentales y organismos estatales, sino que más bien se recurrió a bibliotecas personales, extendiéndose el tiempo dedicado a reunir información.

2 Las bibliotecas y centros de documentación fueron agrupados en las siguientes categorías: (a) universidades, (b) centros de documentación de organismos no gubernamentales, (c) organismos gubernamentales, (d) organismos internacionales, (e) bibliotecas públicas y personales y (f) centros de investigaciones. 
La sistematización da cuenta de la existencia de un número significativo de investigaciones (49) en distintas temáticas relacionadas con salud sexual y reproductiva. Del total en regiones (46), 17 son tesis universitarias de pregrado y de posgrado, 13 realizadas por organismos gubernamentales, tres por organismos no gubernamentales, dos por entidades asociadas, una sin clasificación institucional y diez realizadas por universidades. También se incluyen algunas que, aunque no se refieren exclusivamente a estas temáticas, entregan aportes significativos para esta recopilación ${ }^{3}$.
Las instituciones que realizaron estudios en salud sexual y reproductiva y pueblos indígenas fueron fundamentalmente organismos gubernamentales y universidades. Las realizadas por los primeros son 13 , concentrándose la mayoría en la Primera y la Novena regiones, con un total de cuatro investigaciones cada una. Por su parte, las universidades efectuaron un total de diez investigaciones -también en la Primera y la Novena regiones-y 17 tesis. Por lo tanto, la mayoría de los estudios se han realizado en universidades (27).

Número de investigaciones por instituciones y región

\begin{tabular}{|l|c|c|c|c|c|c|c|c|c|}
\hline Regiones & I & II & V & RM & VIII & IX & X & Total \\
\hline Tipos de institución & 4 & 1 & 1 & 2 & - & 4 & 1 & 13 \\
\hline Gubernamentales & 2 & - & 1 & - & - & - & & 3 \\
\hline Ong's & 4 & - & - & - & - & 4 & 2 & 10 \\
\hline Universidades & 11 & $1^{*}$ & $2^{* *}$ & - & 1 & 2 & - & 17 \\
\hline Tesis universitarias & - & - & - & - & - & 2 & - & 2 \\
\hline Asociadas & - & - & - & 1 & - & - & - & 1 \\
\hline Otras & 21 & 2 & 4 & 3 & 1 & 12 & 3 & 46 \\
\hline Total & & - & & & & & 3 \\
\hline
\end{tabular}

* La tesis pertenece a la Universidad de Chile con sede en Santiago.

** Una de las tesis es de la Universidad de Barcelona.

Respecto de las tesis universitarias, la mayoría se concentra en la Primera Región (11) y fueron realizadas principalmente en carreras de salud: obstetricia y tecnología médica. Estas tesis tienen en su mayoría un enfoque biomédico y carecen de una perspectiva de género y derechos.

Número de tesis por carrera y región

\begin{tabular}{|c|c|c|c|c|c|c|c|c|}
\hline Regiones & $\mathrm{I}$ & II & $\mathrm{V}$ & $\mathrm{RM}$ & VIII & IX & $\mathrm{X}$ & Total \\
\hline \multicolumn{9}{|l|}{ Tesis universitarias } \\
\hline Antropología & - & 1 & 1 & - & - & - & - & 2 \\
\hline Psicología & 1 & - & - & - & - & - & - & 1 \\
\hline Trabajo Social & - & - & - & - & - & 1 & - & 1 \\
\hline Obstetricia & 6 & - & - & - & 1 & - & - & 7 \\
\hline Tecnología médica & 4 & - & - & - & - & - & - & 4 \\
\hline Sociología & - & - & - & - & - & 1 & - & 1 \\
\hline Doctorado & - & - & 1 & - & - & - & - & 1 \\
\hline Total & 11 & 1 & 2 & - & 1 & 2 & - & 17 \\
\hline
\end{tabular}

La mayoría de las investigaciones encontradas tienen un carácter biomédico. No obstante, en las regiones I y IX destacan estudios sobre concepciones y prácticas en sexualidad y salud reproductiva de los pueblos

3 Santa Coloma, María Eugenia. "Transición demográfica y social: el caso Rapa Nui” y Citarilla, Luca. Medicinas y Culturas en la Araucanía”. aimara y mapuche. Este tipo de estudios es escaso y se han realizado sobre todo en contextos rurales, pese a la alta concentración de población originaria que vive en ciudades.

Sorprende la escasez de investigaciones en las regiones II, VIII y Metropolitana, pues allí se ubican diversas 
instituciones gubernamentales, universidades y organizaciones sociales relacionados con la temática de los pueblos indígenas, a la par de contar con una alta concentración de población originaria.

Las metodologías utilizadas en las investigaciones se clasificaron en relación con los propios datos señalados en cada una de ellas. Algunas de ellas describieron las técnicas de recolección de información usadas. Hay que destacar que utilizan mayoritariamente metodologías cuantitativas; sin embargo, en años recientes, en la investigación nacional e internacional en sexualidad y reproducción para población general (no indígena) hay una tendencia a la complementación metodológica entre los enfoques cuantitativo y cualitativo. Esta tendencia es también observable, aunque de manera reciente, entre las investigaciones recopiladas. $\mathrm{La}$ incorporación de lo cualitativo posibilita comprender fenómenos que son interpretados de manera distinta por cada pueblo indígena.

Tipo de metodología de las investigaciones por región

\begin{tabular}{|c|c|c|c|c|c|c|c|c|}
\hline Regiones & I & II & $\mathrm{V}$ & $\mathrm{RM}$ & VIII & IX & $\mathrm{X}$ & Total \\
\hline \multicolumn{9}{|l|}{ Tipos de metodología } \\
\hline Cualitativa & 3 & 3 & 4 & - & - & 4 & 2 & 16 \\
\hline Cuantitativa & 13 & - & - & 3 & 1 & 5 & 1 & 23 \\
\hline Cualitativa y cuantitativa & 4 & - & - & - & - & 3 & - & 7 \\
\hline Sin dato & 1 & - & - & - & - & - & - & 1 \\
\hline Total & 20 & 3 & 4 & 3 & 1 & 11 & 3 & 46 \\
\hline
\end{tabular}

Las fuentes de financiamiento provienen en su mayoría de organismos gubernamentales, lo cual es, a nuestro juicio, coherente con la escasa presencia en el país de fuentes de financiamiento internacional o independiente, y destacable porque no existe una política pública en salud sexual y reproductiva para pueblos originarios con fondos específicos para la investigación. De las 22 investigaciones que incluían información sobre este aspecto, 12 fueron financiadas por organismos gubernamentales, seis por agencias internacionales y cinco con fondos universitarios ${ }^{4}$.

En su mayoría, las investigaciones han sido publicadas en algún formato. Se consideraron ocho tipos de publicaciones: actas de congreso, libros, artículos en libros, documentos de trabajo, artículos en revistas, textos institucionales (principalmente folletos y CD $\mathrm{ROM}$ ), páginas Web e informes internos de proyectos.
Es importante señalar que algunas han generado más de una publicación.

\begin{tabular}{|l|l|}
\hline Actas de congresos & 12 \\
\hline Libros & 7 \\
\hline Textos institucionales & 6 \\
\hline Artículos en revistas & 3 \\
\hline Informes internos & 3 \\
\hline Artículos en libros & 2 \\
\hline Documentos de trabajo & 2 \\
\hline Página Web & 1 \\
\hline
\end{tabular}

Los temas que presentan más investigaciones son "embarazo y parto" y se concentran principalmente en la I Región. Le siguen "enfermedades de transmisión sexual (ETS)” y "calidad de la atención”, también mayoritariamente en esa región. Las temáticas menos abordadas fueron "menopausia", "amenorrea" y "amamantamiento".

4 De las fuentes gubernamentales, tres estudios fueron financiados por FONDECYT, cinco por el Ministerio de Salud, uno por el Ministerio de Cooperación y Planificación y dos por el Servicio Nacional de la Mujer. Las agencias internacionales que figuran financiando las investigaciones son: UNFPA, PNUD, Special Programme of Research Development and Research Training in Human Reproduction (WHO), Cooperación Italiana, Agencia de Cooperación Alemana (GTZ) y Fundación Ford. De siete investigaciones que no informan su fuente, suponemos que han tenido financiamiento del Ministerio de Salud. 
Número de temáticas de investigación por región

\begin{tabular}{|c|c|c|c|c|c|c|c|}
\hline \begin{tabular}{|l|} 
Regiones \\
\end{tabular} & $\mathrm{I}$ & II & $\mathrm{V}$ & $\mathrm{RM}$ & IX & $\mathrm{X}$ & Total \\
\hline \multicolumn{8}{|l|}{ Temas de investigación } \\
\hline Interculturalidad & 1 & 1 & - & - & 1 & - & 3 \\
\hline Epidemiología & 1 & - & - & 1 & 2 & - & 4 \\
\hline Embarazo y parto & 7 & - & - & 1 & 3 & 1 & 12 \\
\hline Amenorrea & - & - & - & - & 1 & - & 1 \\
\hline Amamantamiento & - & - & - & - & 1 & - & 1 \\
\hline Regulación de la fertilidad & 4 & 1 & - & - & 1 & - & 1 \\
\hline Aborto & 1 & 1 & - & - & - & - & 2 \\
\hline Maternidad y paternidad & - & 1 & - & - & - & - & 1 \\
\hline VIH/Sida & - & - & 1 & - & 1 & - & 2 \\
\hline Menopausia & - & - & - & 1 & - & - & 1 \\
\hline Enfermedades de transmisión sexual & 3 & - & - & - & - & - & 3 \\
\hline \begin{tabular}{|l|} 
Violencia Doméstica e intrafamiliar \\
\end{tabular} & 1 & - & - & - & 1 & 1 & 3 \\
\hline Derechos sexuales y reproducivos & 1 & - & - & - & - & 1 & 2 \\
\hline Calidad de atención & 3 & - & - & 2 & 1 & 2 & 8 \\
\hline Sexualidad & 2 & 1 & 1 & - & 1 & 1 & 6 \\
\hline Sexualidad juvenil & - & - & 1 & - & 1 & - & 2 \\
\hline Fecundidad & - & - & - & - & 3 & 1 & 4 \\
\hline Cuerpo & 2 & - & - & - & 1 & - & 3 \\
\hline Control ginecológico & 1 & - & - & 2 & - & - & 3 \\
\hline
\end{tabular}

Respecto de la calidad de la atención en salud sexual y reproductiva, pese a que no encontramos investigaciones de satisfacción de los usuarios, varias informan que las mujeres indígenas se quejan por la escasa información que reciben sobre los procedimientos a que son sometidas, algunos en contradicción con su cultura, como es el caso de baños con agua fría en el parto, al igual que la posición de la mujer al momento de parir 5 . De las 17 tesis, ocho se refieren a enfermedades de transmisión sexual, principalmente en la I Región, y se vinculan con Obstetricia y Tecnología Médica; cinco a embarazo y parto, y tres a puerperio, calidad de la atención y sexualidad respectivamente. Estas últimas concentradas en las regiones Primera, Segunda y Quinta.

La perspectiva de género, entendida como una categoría que otorga significado a la experiencia de salud y enfermedad en hombres y mujeres, ha sido utilizada sólo en siete de las 49 investigaciones. De éstas, tres se ubican en la Primera Región, dos son parte de los estudios realizados por organizaciones no gubernamen- tales y una la llevó a cabo la Universidad de Tarapacá en conjunto con el Taller de Estudios Andinos (TEA). Le sigue la Segunda Región con una realizada por el Servicio Nacional de la Mujer (SERNAM). Dentro de los estudios nacionales, sólo una investigación ha incorporado perspectiva de género, realizada por el Centro de Estudios de Género de la Facultad de Ciencias Sociales de la Universidad de Chile para la Comisión Nacional del SIDA (CONASIDA) y el Ministerio de Salud (MINSAL).

Además, se encontraron tres investigaciones de carácter nacional: dos de ellas financiadas por organizaciones gubernamentales - por el Ministerio de Salud y por la OPS-y otra no contiene información sobre fuentes de financiamiento. Dos han utilizado tanto metodologías cualitativas como cuantitativas, y una sólo cualitativa. Respecto de las publicaciones, una ha sido publicada en actas de congreso y en revista. Las temáticas abordadas se concentran en "calidad de atención" (3) y "embarazo y parto" (2).

5 Seminario Mujer, Cultura y Salud en los Andes. Arica: 2002. 
Número de temáticas estudios de carácter nacional

\begin{tabular}{|l|c|}
\hline Temas de investigación & Número \\
\hline Mortalidad materna & 1 \\
\hline Fecindidad & 1 \\
\hline Embarazo y parto & 2 \\
\hline Regulación de la fertilidad & 1 \\
\hline Enfermedades de transmisión sexual & 1 \\
\hline VIH/SIDA & 1 \\
\hline Calidad de atención & 3 \\
\hline Sexualidad & 1 \\
\hline Violencia & 1 \\
\hline Cuerpo & 1 \\
\hline Políticas Públicas & 1 \\
\hline
\end{tabular}

\section{Conclusión}

La pertinencia étnica en las investigaciones en salud sexual y reproductiva en pueblos indígenas se plantea como un dilema ético actual, debido a la necesidad de incorporar en alguno de los procesos investigativos las particularidades de los pueblos indígenas de nuestro país. Ejemplos de esa carencia se pueden encontrar en:

1. El uso de técnicas de recolección de información que no consideran sus creencias y tradiciones, aplicando encuestas en espańol y planteando la dificultad de la traducción de conceptos que no existen en todas las lenguas.

2. La escasa relación entre el estudio de algunas temáticas en determinadas zonas del país y las necesidades reales de la población indígena.

3. La mayoría de las investigaciones son llevadas a cabo desde una perspectiva exclusivamente biomédica. Incorporar perspectivas socioculturales en salud sexual y reproductiva puede ayudar a vislumbrar factores de riesgo y protectores intraculturales, y favorecer la elaboración de investigaciones, la adaptación de técnicas de recolección de información y las formas de devolución de resultados a grupos culturales diversos.

4. No se encuentra descrito en las investigaciones la devolución de los resultados de los estudios a las comunidades donde éstas fueron realizadas.

Frente a la necesidad de conocer la situación de salud sexual y reproductiva de la población indígena en nuestro país, resulta especialmente preocupante la falta de datos respecto de frecuencia, recurrencia y/o incidencia del uso de métodos anticonceptivos y atenciones de embarazo, parto y puerperio, esterilizaciones voluntarias, cáncer cervicouterino y de mamas, VIHSIDA/ETS, entre otros. Estos datos podrían permitir la comparación con la población no indígena para diseñar posibles investigaciones, identificar brechas en salud, líneas de acción y políticas públicas.

\section{Bibliografía}

Alarcón AM, Vidal A, Neira J. Salud intercultural: elementos para la construcción de sus bases conceptuales. Revista Médica de Chile 2003; 131: 1061-1065.

CEDEMU Sentando bases para el desarrollo de un modelo intercultural en salud sexual y reproductiva entre las mujeres aymará del norte de Chile: un estudio de epidemiología intercultural, balance del proceso. Arica: CEDEMU; 2001-2002.

Citarella L, (comp.) Medicinas y culturas en la Araucanía. Santiago de Chile: Editorial Sudamericana; 2000.

Enlace Continental de Mujeres Indígenas en CEPAL. Reunión de la Comisión Económica para América Latina, CEPAL, San Juan 28 de junio 2004. (Sitio en Internet) Disponible en: www.radiofeminista.net Último Acceso en mayo de 2005.

Gobierno de Chile. Nuevo trato con los pueblos indigenas. Derechos indigenas, desarrollo con identidad y diversidad cultural. Santiago de Chile: 2004. 
Investigaciones en salud sexual y reproductiva de pueblos indígenas en Chile y la ausencia de pertinencia étnica - C. Dides y M. Pérez

Ibacache J, Oyarce AM. Reflexiones para una politica intercultural en salud. Puerto Saavedra, Chile: Primer encuentro Nacional de salud y Pueblos Indígenas; 1996.

Informe de la Conferencia Internacional sobre la Población y el Desarrollo. El Cairo, 5 a 13 de septiembre de 1994 . (Sitio en Internet) Disponible en: www.cinu.otg.mx/temas/desarrollo/dessocial/población/icpdf1994.htm Último acceso en septiembre de 2004 .

Ministerio de Salud MINSAL, Gobierno de Chile. Programa Especial de Pueblos Indigenas. (Sitio en Internet) Disponible en: www.minsal.cl Último acceso en noviembre de 2004.

Pérez MS. Reflexiones sobre la medicina tradicional y medicina occidental en Chile a través del proceso de salud-enfermedad. Anuario de Escuela de Postgrado. Santiago de Chile: Facultad de Filosofía y Humanidades; 2003.

Pérez MS, Dides C. Salud, Sexualidady Reproducción. Sistematización de investigaciones y experiencias en pueblos indígenas en Chile 1990-2004. Santiago de Chile: CORSAPS -UNFPA; 2005.

Recibido: 12 de julio de 2007.

Aceptado: 23 de agosto de 2007. 